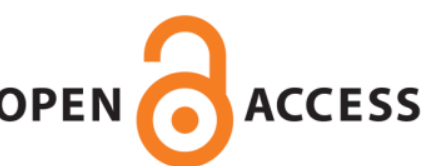

UWS Academic Portal

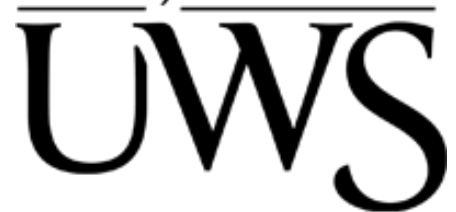

\title{
Effects of severe hallux valgus on metatarsal stress and the metatarsophalangeal loading during balanced standing: A finite element analysis
}

Zhang, Yan; Awrejcewicz, Jan; Szymanowska, Olga; Shen, Siqin; Zhao, Xiaoxue; Baker, Julien S.; Gu, Yaodong

Published in:

Computers in Biology and Medicine

DOI:

10.1016/j.compbiomed.2018.04.010

E-pub ahead of print: 16/04/2018

Document Version

Peer reviewed version

Link to publication on the UWS Academic Portal

Citation for published version (APA):

Zhang, Y., Awrejcewicz, J., Szymanowska, O., Shen, S., Zhao, X., Baker, J. S., \& Gu, Y. (2018). Effects of severe hallux valgus on metatarsal stress and the metatarsophalangeal loading during balanced standing: $A$ finite element analysis. Computers in Biology and Medicine, 97, 1-7.

https://doi.org/10.1016/j.compbiomed.2018.04.010

\section{General rights}

Copyright and moral rights for the publications made accessible in the UWS Academic Portal are retained by the authors and/or other copyright owners and it is a condition of accessing publications that users recognise and abide by the legal requirements associated with these rights. 


\section{Accepted Manuscript}

Effects of severe hallux valgus on metatarsal stress and the metatarsophalangeal loading during balanced standing: A finite element analysis

Yan Zhang, Jan Awrejcewicz, Olga Szymanowska, Siqin Shen, Xiaoxue Zhao, Julien

S. Baker, Yaodong Gu

PII: $\quad$ S0010-4825(18)30085-4

DOI: $\quad$ 10.1016/j.compbiomed.2018.04.010

Reference: $\quad$ CBM 2935

To appear in: Computers in Biology and Medicine

Received Date: 7 February 2018

Revised Date: 14 April 2018

Accepted Date: 14 April 2018

Please cite this article as: Y. Zhang, J. Awrejcewicz, O. Szymanowska, S. Shen, X. Zhao, J.S. Baker, Y. Gu, Effects of severe hallux valgus on metatarsal stress and the metatarsophalangeal loading during balanced standing: A finite element analysis, Computers in Biology and Medicine (2018), doi: 10.1016/ j.compbiomed.2018.04.010.

This is a PDF file of an unedited manuscript that has been accepted for publication. As a service to our customers we are providing this early version of the manuscript. The manuscript will undergo copyediting, typesetting, and review of the resulting proof before it is published in its final form. Please note that during the production process errors may be discovered which could affect the content, and all legal disclaimers that apply to the journal pertain. 
1

2

3

4 Yan Zhang ${ }^{1,2}$, Jan Awrejcewicz ${ }^{2}$, Olga Szymanowska ${ }^{2}$, Siqin Shen ${ }^{1}$, Xiaoxue Zhao ${ }^{1}$, Julien S

$5 \quad$ Baker $^{3}$, Yaodong $\mathrm{Gu}^{1}$

61 Faculty of Sports Science, Ningbo University, China

72 Department of Automation, Biomechanics and Mechatronics, The Lodz University of

8 Technology, Poland

93 Institute of Clinical Exercise and Health Science, University of the west of Scotland, UK

10

11

12

13

14

15

16

\section{during balanced standing: A finite element analysis}

\section{Effects of severe hallux valgus on metatarsal stress and the metatarsophalangeal loading}


17 Abstract

18 The internal stress of the human foot enables efficient parametric evaluation of structural and functional impairments associated with foot deformities, such as hallux valgus (HV). However, the status of the internal stress of such a deformed foot remains insufficiently addressed due to the difficulties and limitations of experimental approaches. This study, using finite element (FE) methodology, investigated the influence of severe HV deformity on the metatarsal stress and the metatarsophalangeal (MTP) joint loading during balanced standing. FE models of a normal foot and a severe HV were constructed and validated. Each FE model involves 28 bones and various cartilaginous structures, ligaments, and plantar fascia, as well as encapsulated soft tissue. All the materials except for the encapsulated soft tissue were considered isotropic and linearly elastic, while the encapsulated soft tissue was set as nonlinear hyperelastic. Hexahedral elements were assigned to the solid parts of bones, cartilage, and the encapsulated soft tissue. Link elements were assigned to ligaments and plantar fascia. A plate was created for simulating ground support. A vertical force of a half-body weight was applied on the bottom of the plate for simulating balanced standing loading. The superior surfaces of the encapsulated soft tissue, distal tibia, and distal fibula were fixed. Stress distribution in the metatarsals, contact pressure, and force at the MTP joints were comparatively analysed. Compared to the normal foot, the HV foot showed higher stress concentration in the metatarsals but lower magnitude of MTP joint loading. In addition, the region with high contact pressure at the first MTP joint shifted medially in the HV foot. Knowledge of this study indicates that patients with severe HV deformity are at higher risk of metatarsal injuries and functional impairment of the MTP joints while weight bearing.

Keywords: Finite element, Severe hallux valgus, Metatarsal stress, Metatarsophalangeal loading

\section{Introduction}


Hallux valgus (HV) is a common foot deformity characterized by progressive lateral deviation of the hallux with medial deviation of the first metatarsal. Indexes of hallux valgus angle (HVA) and intermetatarsal angle (IMA) are commonly used radiological measurements for assessing the degree of deformity, which is classified as mild (HVA: $15^{\circ}-20^{\circ}$; IMA: $9^{\circ}-11^{\circ}$ ), moderate (HVA: $20^{\circ}-40^{\circ}$; IMA: $11^{\circ}-16^{\circ}$ ), and severe (HVA: $>40^{\circ}$; IMA: $>16^{\circ}$ ) [1]. Increasing HV severity may cause significant health-related problems, such as metatarsalgia [2], balance deficits [3], and increase in risk of falling in older people [4].

Because the metatarsal bones act as a unit in the forefoot area to provide a broad plantar surface for load bearing, the majority of research on biomechanical consequences of $\mathrm{HV}$ deformity have referred to the alternation of forefoot loading recorded by plantar pressure measurements. HV deformity has been reported as resulting in increased forefoot plantar pressure, with debate about which region the change occurs in. Some studies have suggested that the peak pressure increased significantly under the first and second metatarsals in HV feet compared with that in normal feet $[5,6,7,8]$. There is contrary evidence that HV deformity causes increased load on the lateral metatarsals [2, 9]. Koller et al. [10] assessed the plantar pressure of HV feet of different grades and found a positive correlation between HV grade and the peak pressure of the fifth metatarsal head. On the other hand, changes in the loading state of the inner foot are poorly explored.

As a result of the intrinsic difficulties and limitations of conventional experimental methods, the internal loading of cartilaginous and bony structures cannot be measured directly. Finite element (FE) methodology is increasingly used to simulate the mechanical responses of biological systems via a numerical model simulating complicated boundary and loading conditions. The FE model is capable of predicting the internal stress in the foot complex and eventually interpreting potential risks associated with foot deformities. Through reducing the stiffness of ligaments, Wong et al. [11] developed a FE foot model of first ray hypermobility which presented higher resultant metatarsocuneiform joint force along with an abrupt change in medial-lateral direction. They interpreted failure of the joint to accommodate the change of joint 
71 force as possible deviation of the first metatarsal from normal alignment, possibly causing

72 metatarsus primus varus. Extrinsic factors, such as high-heeled shoes, have been reported to be

73 responsible for the occurrence of foot disorder. Using FE method, Yu et al. [12] suggested that

74 the increased von Mises stress over the lateral and dorsal regions of the first

75 metatarsophalangeal (MTP) joint during prolonged high-heeled standing may induce the

76 development of HV deformity. Wong et al. [13] assumed HV deformity to be a normal foot

77 with hypermobility and manifested that the arthrodesis of the first metatarsocuneiform is a

78 useful treatment for functional restoration of $\mathrm{HV}$ based on the increased compressive stress in

79 the first metatarsal. Furthermore, a subject-specific FE model of HV foot was developed

80 recently to compare the effects of different surgical fixation methods on HV treatment by

81 predicting the peak von Mises stress and compression stress of the distal fragment of the first

82 metatarsal [14].

83 Information on stress distribution of the internal structures is useful in enhancing the

84 understanding of podiatric biomechanics and may contribute to surgical treatments. In this study,

85 FE foot models of a normal subject and a severe hallux valgus (HV) patient were developed to

86 evaluate the metatarsal stress and the MTP joint contact pressure under a balanced standing

87 condition. It was hypothesized that metatarsal stress would increase while the MTP joint contact

88 pressure would decrease in the severe HV foot.

\section{Material and methods}

$91 \quad 2.1$ Model construction

92 This study has been approved by the Human Ethics Committee of Ningbo University 93 (ARGH20170213). HVA and IMA for the HV foot were $48.07^{\circ}$ and $16.17^{\circ}$, respectively; and 94 those for the normal foot were $22.82^{\circ}$ and $14.14^{\circ}$, respectively (Figure 1). The three95 dimensional models of the normal foot and the HV foot were reconstructed from computer tomography (CT) images of a 26-year old female (height: $165 \mathrm{~cm}$; weight: $51 \mathrm{~kg}$ ) and a 37 -year 
97 old female (height: $163 \mathrm{~cm}$; weight: $52 \mathrm{~kg}$ ) respectively. Both participants had no other

98 musculoskeletal pathology, pain, or lower limb injury or surgery within the past 12 months.

99 The coronal CT images were obtained with a space interval of $2 \mathrm{~mm}$ without weight-bearing

100 from the left foot. The two-dimensional images were segmented using MIMICS 16.0

101 (Materialise, Leuven, Belgium) to obtain the three-dimensional model of the bone tissue and the

102

103

104

105

106

107

108

109

110

111

112

113

114

115

116

117

118

119

120

121

122

123 encapsulated soft tissue. The uneven surface of the geometries of the bony components and the soft tissue were smoothed using Geomagic Studio 2013 (Geomagic, Inc., Research Triangle Park, North Carolina, USA). Each surface component was then imported into Solidworks 2016 (SolidWorks Corporation, Massachusetts, USA) individually to form a solid part. To model a cartilaginous structure, a solid volume was created between the adjacent surfaces of two bones, and the bones were then subtracted from the solid volume. All bones and cartilage were subtracted from the full volume of soft tissue to create the encapsulated soft tissue. The numerical foot model consisted of 28 bony segments, including tibia, fibula, talus, calcaneus, cuboid, navicular, three cuneiforms, five metatarsals, and 14 phalanges. Link elements that have tension-only capability were used to model ligaments. A total number of 76 ligaments and five plantar fascia were included and defined by connecting attaching points on corresponding bones with straight line structures. The attaching regions were determined by reference to an anatomy book [15] and the attaching points were approximately close to the geometrical centre of the attaching regions. The plantar fascia consisted of five separate rays connecting the insertions between the calcaneus and the proximal phalanges. The plantar ligaments and the spring ligament were represented by two separate rays, and small ligaments were represented by a single ray. HyperMesh 13.0 (Altair Engineering Inc., Hyperworks, America) was used for mesh generation. Each bony and cartilaginous component and the encapsulated soft tissue were partitioned into sub-volumes and then assigned hexahedral elements for each volume. The mesh size for both models was $4.5 \mathrm{~mm}$ for the encapsulated soft tissue, $3 \mathrm{~mm}$ for the bones, and 2.5 $\mathrm{mm}$ for the cartilaginous structures. Mesh sensitivity tests were conducted on the whole foot model under conditions of balanced standing by gradually reducing the mesh sizes. An 
acceptable mesh generation is determined as the deviation of resultant peak equivalent stress (PES) in the first metatarsal, and the calcaneus was within 5\% when further reducing mesh sizes. For the normal foot model, the deviation of PES in the first metatarsal and the calcaneus were $1.56 \%$ and $2.24 \%$, respectively; and for the HV foot model, the deviation data were $2.38 \%$ and $3.82 \%$, respectively. The total number of elements for the normal foot model was 195,237 and that for the HV foot model was 180,885. The FE package ANSYS Workbench 17.0 (ANSYS, Inc., Canonsburg, USA) was used for subsequent analysis. This software provides automated contact detection for assemblies. Using algorithms based on surface proximity, it is able to create possible contact pairs. Surface-to-surface contact was used to simulate the interaction of the surfaces of the cartilaginous and bony structures. The contact between bone and cartilaginous surfaces was assumed as frictionless [16, 17]. All the bones and cartilage were bonded to the encapsulated soft tissue.

All the materials except for the encapsulated soft tissue were considered isotropic and linearly elastic with properties obtained from previous literature $[17,18]$. The two material constants of Young's modulus E and Poisson's ratio $v$ were assigned to represent the elasticity. The encapsulated soft tissue was set as nonlinear hyperelastic material which was defined as a Moonley-Rivlin model. The element types and material properties used are listed in Table 1 and Table 2

\subsection{Load and boundary conditions}

A balanced standing condition was considered for the FE analysis. The superior surfaces of the encapsulated soft tissue, distal tibia, and distal fibula were fixed (Figure 2). A plate was created assigned with an elastic property to model the ground support (Table 1). The plate was allowed to move freely only in the vertical direction. A vertical ground reaction force (GRF) of a halfbody weight ( $255 \mathrm{~N}$ for normal foot; $260 \mathrm{~N}$ for $\mathrm{HV}$ foot) was applied at the inferior surface of the plate. The interaction between the foot and the plate was simulated as contact surface with a coefficient of friction of 0.6 [12]. Five equivalent force vectors representing the Achilles tendon 
151 force were applied on the posterior extreme of the calcaneus. The force of the Achilles tendon

152 (128 $\mathrm{N}$ for normal foot; $130 \mathrm{~N}$ for $\mathrm{HV}$ foot) was estimated as $50 \%$ of the load applied on the

153 foot while balanced standing [25].

154

155

2.3 Model validation

156

The numerical model was validated by comparing plantar pressure obtained from computational simulation in FE software and experimental measurement by a Novel emed pressure platform (Novel, Munich, Germany) in a standing position. The measurement was performed on the same subject who had volunteered for the medical image scanning. Both subjects were asked to stand still on the pressure platform for five seconds. Data from the middle three seconds were selected and averaged. The validated models were then used for FE analysis on forefoot biomechanics.

\section{Result}

\subsection{Model validation}

Figure 3 shows the comparison of predicted plantar pressure (b, d) with experimental results (a, c). Pressure distribution from the FE result was generally consistent with the experimental result. Also, a good match between peak pressure value and location were found for both models (Figure 3). For the normal foot model, the peak pressure from prediction and measurement was $0.141 \mathrm{MPa}$ and $0.135 \mathrm{MPa}$, respectively, and for the $\mathrm{HV}$ foot the values were $0.144 \mathrm{MPa}$ and $0.137 \mathrm{MPa}$, respectively. The second highest pressure was located under the first metatarsal head for both models. The predicted pressure of the normal foot model and HV foot model was 0.089 $\mathrm{MPa}$ and $0.122 \mathrm{MPa}$, respectively, and the corresponding measured pressure was 0.093 MPa and 0.127 MPa, respectively. This suggests that the models are valid for further simulation. 
177 The von Mises stress in metatarsals generally increased due to HV deformity, as shown in

178 Figure 4. For both the normal and HV foot, the third metatarsal experienced the highest von

179 Mises stress concentration, followed by the second, fourth, and first metatarsals, and the lowest

180 stress level existed at the fifth metatarsal. Compared to the normal foot, medial and lateral

181 forefoot sustained a larger percentage of von Mises stress in the HV foot. The severe HV foot

182 showed pronounced increases in von Mises stress at the fifth (55\%), first (44\%), and fourth

183 (40\%) metatarsals. For the third metatarsal, it was $4.12 \mathrm{MPa}$ in the normal foot and increased to

184 4.24 MPa due to HV deformity. However, the stress concentration at the second metatarsal

185 decreased (less than 3\%) in the severe HV foot.

\subsection{MTP joint contact pressure}

The severe HV foot showed lower MTP joint contact pressure, especially at the second to fifth MTP joints (Figure 5). Contact pressure at the first MTP of the severe HV foot (0.28 MPa) was slightly lower than that of the normal foot $(0.3 \mathrm{MPa})$. Figure 6 illustrates the apparently different locations of high contact pressure (Unit: MPa) at the first MTP joint between the severe HV foot and the normal foot. For the normal foot, the maximum pressure is located at central bottom of the contact surface, while for the severe HV footit transfers medially so that it is located at medial bottom of the contact surface.

\subsection{MTP joint force}

197 The MTP joint force was defined as the reaction force imposed by the first metatarsal on the proximal/posterior surface of the cartilage between the first metatarsal and the proximal phalanx.

The component MTP force was dominant in the anterior-posterior direction $(4.58 \mathrm{~N}$ for the severe HV foot and $8.67 \mathrm{~N}$ for the normal foot), while it only accounted for $7.1 \%$ in the severe $\mathrm{HV}$ foot and $10 \%$ in the normal foot in medial-lateral and superior-inferior directions. The resultant MTP force of the severe HV foot was nearly 50\% less than that of the normal foot (Figure 7). 


\section{Discussion}

206

The development of a comprehensive computational model of the human foot was suggested for research focusing on podiatric biomechanics, so as to overcome the intrinsic limitations of in vivo experiments for further understanding of foot problems [26]. This study explored the effects of severe hallux valgus on metatarsal stress and on metatarsophalangeal joint loading while balanced standing using the FE method. The development of patient-specific models is advocated for foot problems with distinct structural deformity [27, 28]. Knowledge of this study would provide information for medical treatment of HV and subject-specific footwear design.

Three-dimensional FE models were validated by plantar pressure measurement. This method was commonly used in the validation of a FE foot model [18]. In general, the predicted plantar pressure distribution and peak pressure were comparable to the experimental measurements. The material properties of the bony, cartilaginous, ligamentous, and encapsulated-soft-tissue components were considered consistent between the normal foot and the deformed foot. Many studies have developed normal-foot FE models or a pathological model from the normal one to investigate internal foot biomechanical performance under various conditions [28]. In an attempt to promote the understanding of $\mathrm{HV}$ pathomechanics and to improve the effectiveness of treatments, a FE model of the first ray has been constructed for predicting mechanical consequences of mild HV deformity [29]. An entire foot model featured with skeletal deformity of severe HV was also developed and validated in this study to provide potentials for research purposes and clinical application. Previous FE models relied heavily on the tetrahedral elements for meshing bones, cartilage, and soft tissue on account of the effort attributed to developing a hexahedral mesh of irregular geometry. In theory, the hexahedral element, due to its nature of having relatively more degrees of freedom, is able to provide more accurate FE analysis [30, 31]. Although one study modelling the proximal femur showed that tetrahedral and hexahedral 
231 elements presented nearly the same resultant peak von Mises stress, the CUP time was

232 significantly less when using hexahedral elements, indicating a more efficient computational

233 foundation of the hexahedral element mesh scheme. The bony and cartilaginous components

234 and the encapsulated soft tissue were meshed as hexahedral elements in this study, which was

235 proposed to be utilized for better comparison of FE predicted results by $\mathrm{Yu}$ et al. [12]. To improve the mesh quality and the percentage of hexahedral elements, the individual bony and cartilaginous components and the soft tissue were partitioned prior to mesh generation in HyperMesh 13.0.

\subsection{Metatarsal von Mises stress}

The five metatarsal bones have an important function with respect to weight bearing. Previous experimental results advocated medial or lateral shift of forefoot plantar pressure due to HV deformity. This study predicted obviously higher plantar pressure under the first metatarsal head in the HV foot model than in the normal foot model, which is consistent with the outcomes measured by Martínez-Nova et al. [8]. As to the inner stress, the von Mises stress is often considered as one predictor for stress failure of foot bone which has been widely used for evaluating the risk of long-term pathological changes. Cheung et al. [32] noted that the intensified stress at central metatarsals and dorsal calcaneocuboid joint junction may lead to midfoot pain. This study found higher peak von Mises stress in the metatarsals, except for the second one for the severe HV foot, indicating an increasing risk of stress or fatigue failure. As balanced standing is the most common and basic behaviour in daily life, it can be speculated that the increased metatarsal stress may cause metatarsalgia while sustaining weight bearing. The most obvious increasing of von Mises stress at the fifth metatarsal indicates that the fifth metatarsal is more susceptible to injury for patients with severe HV. The first metatarsal should be expected to avoid high stress during weight bearing in cases of first ray deformity, however, the predicted stress at the first metatarsal for the severe HV foot was $44 \%$ higher than that of the normal foot. This is very likely to be associated with medial arch collapse, which is often thought to supervene with hallux valgus [33]. The crossover second toe also commonly occurs 
258

259

260

261

262

263

264

265

266

267

268

269

270

271

272

273

274

275

276

277

278

279

280

281

282

283

284

in the presence of hallux valgus as shown in Figure 1 (a) [34]. The abnormal alignment of second ray seems a possible factor leading to the slightly decreased stress of the second metatarsal. It is important to note that the crossover second toe is not a definite condition accompanied by hallux valgus, indicating that the crossing toes may hinder the generalization of the findings.

\subsection{MTP joint loading}

The contact pressure of MTP joints was lower in the severe HV foot, especially for the second to fifth MTPs. Similarly, the resultant joint force of the first MTP also showed lower magnitude than that of the normal foot. The decreased joint loading may imply the impairment of load bearing and transfer function of the first MTP joint in gait. In agreement with this speculation, Zhang et al. [29] found weakened windlass mechanism in the HV foot during initial push-off. As to the contact pressure of the first MTP joint, there is only a slight decrease for the severe HV foot, while regions with high pressure locate apparently differently between the HV foot and the normal foot. In contrast to the location of the peak contact pressure at central bottom on the cartilage for the normal foot, it shifts to medial bottom for the severe HV foot, which may aggravate the symptom of "painful bunion" which is one of the most common complaints among HV patients [35]. Moreover, the component joint force in the medial-lateral direction presented to be opposite between HV and normal foot. The severe HV foot shows lateral reaction force at the first MTP joint during balanced standing, suggesting that loading of body weight alone could predispose the patient to the risk of developing HV deformity.

\subsection{Limitations}

The FE models in this study were based on some simplifications and assumptions. First, the bone was considered as homogeneous, isotropic, and linear elastic material. There is research reporting that a difference in peak von Mises stress in the femur between isotropic and anisotropic assignment was less than $1.2 \%$ under the loading conditions of double-leg standing. 
285 As a result, it is feasible to consider the bone material as isotropic linear elastics, since this

286 study was conducted under standing load. Additionally, cortical and cancellous bone were not

287 defined, while the bone property was regarded as a weighted average of cortical and trabecular

288 elasticity. Despite lack of validation, the value of elastic modulus (7300 MPa) has been adopted

289 as foot bone material in the majority of studies [36]. Second, the geometry of body representing

290 cartilage was not obtained from actual cartilage structures, as it is difficult to distinguish these

291 in CT images. Third, this study created a representative single-subject model of a severe HV

292 foot (HVA: $22.82^{\circ}$; IMA: $14.14^{\circ}$ ). Foot structure, such as arch height and toe deformity, may

293 vary among HV feet; therefore, the presented data should be considered as a first rough

294 approximation. Lastly, this study considered the skeletal structural difference (i.e., the different

295 angle in Figure 1) as the major cause of mechanical changes. It should be noted that HV is also

296 associated with hypermobility of the first metatarsophalangeal joint, which can be simulated

297 through reducing elastic modulus of forefoot ligaments [11], while the material properties of all

298 corresponding components were assumed consistent between the patient-specific model and the

299 normal model in this study.

300

301

\section{Conclusion}

302

Internal stress is significant for better understanding of foot biomechanics as well as pathology of foot problems related to deformities. This study developed FE models of a normal foot and a severe HV foot to predict the internal metatarsal stress and MTP joint loading during balanced standing. Generally, increased von Mises stress at metatarsals and decreased joint loading at MTP were observed in the HV foot in comparison to the normal foot. Specific to the first MTP joint, the regions with high contact pressure of the severe HV foot exhibited a medial shift. Findings in this study suggest that load bearing may predispose patients to higher risk of metatarsal injuries and the functional impairment of the metatarsophalangeal joints. Further improvement of the FE foot model, including cortical and trabecular bones and inhomogeneous 
311 property assignment, will be conducted to obtain more accurate predictions for better

312 comparison.

313

314 Conflict of interest

315 None declared.

316

317

Acknowledgments

318 The work has been supported by the National Natural Science Foundation of China (81772423),

319 K.C.Wong Magna Fund in Ningbo University and the National Science Centre of Poland under the grant OPUS 9 No. 2015/17/B/ST8/01700 for years 2016-2018.

321

322

\section{Reference}

323

[1] R. Hardy, J. Clapham, Observations on hallux valgus, J. Bone Joint Surg. Br. 33 (1951) 376391.

325

[2] U. Waldecker, Metatarsalgia in hallux valgus deformity: a pedographic analysis, J. Foot Ankle Surg. 41 (2002) 300-308.

327

[3] H.B. Menz, S.R. Lord ,The Contribution of Foot Problems to Mobility Impairment and Falls in Community $\square$ Dwelling Older People, J. Am. Geriatr Soc. 49 (2001) 1651-1656.

[4] H.B. Menz, M.E. Morris, S.R. Lord. Foot and ankle risk factors for falls in older people: a prospective study, J. Gerontol A-Biol. 61 (2006) 866-870.

[5] A.P. Bryant, P. Tinley, K. Singer. Plantar pressure distribution in normal, hallux valgus and hallux limitus feet, The foot, 9 (1999) 115-119.

[6] M. Plank, The pattern of forefoot pressure distribution in hallux valgus, The foot, 5(1995) 814.

[7] K.J. Mickle, B.J. Munro, S.R. Lord, H.B. Menz, J.R. Steele. Gait, balance and plantar pressures in older people with toe deformities, Gait Posture, 34 (2011) 347-351. 
337

338

339

340

341

342

343

344

345

346

347

348

349

350

351

352

353

354

355

356

357

358

359

360

361

362

[8] A. Martínez-Nova, R. Sánchez-Rodríguez, P. Pérez-Soriano, S. Llana-Belloch, A. LealMuro, J.D. Pedrera-Zamorano, Plantar pressures determinants in mild Hallux Valgus, Gait Posture, 32(2010) 425-427.

[9] S.E. Hurn, B. Vicenzino, M.D. Smith, Functional impairments characterizing mild, moderate, and severe hallux valgus, Arthrit. Care Res. 67 (2015) 80-88.

[10] U. Koller, M. Willegger, R. Windhager, A. Wanivenhaus, H.J. Trnka, R. Schuh, Plantar pressure characteristics in hallux valgus feet, J. Orthop Res. 32 (2014) 1688-1693.

[11] D.W.C. Wong, M. Zhang, J. Yu, A.K.L. Leung, Biomechanics of first ray hypermobility: an investigation on joint force during walking using finite element analysis, Med. Eng. Phys. 36 (2014) 1388-1393.

[12] J. Yu, J.T.M. Cheung, Y. Fan, Y. Zhang, A.K.L. Leung, M. Zhang, Development of a finite element model of female foot for high-heeled shoe design, Clin Biomech. 23 (2008) S31-S38.

[13] D.W.C. Wong, Y. Wang, M. Zhang, A.K.L. Leung, Functional restoration and risk of nonunion of the first metatarsocuneiform arthrodesis for hallux valgus: a finite element approach. $\mathbf{J}$ Biomech. 48 (2015) 3142-3148.

[14] R. Mao, J. Guo, C. Luo, Y. Fan, J. Wen, L. Wang, Biomechanical study on surgical fixation methods for minimally invasive treatment of hallux valgus. Med. Eng. Phys. 46(2017) $21-26$

[15] W. Platzer, W. Kahle, Color Atlas and Textbook of Human Anatomy: Locomotor system, Color Atlas and Textbook of Human Anatomy, Fifth ed., New York, 2002.

[16] K. Athanasiou, G.T. Liu, L.A. Lavery, D.R. Lanctot, R.C. Schenck Jr., Biomechanical topography of human articular cartilage in the first metatarsophalangeal joint, Clin. Orthop. Relat R. 348 (1998) 269-281.

[17] Wu L. Nonlinear finite element analysis for musculoskeletal biomechanics of medial and lateral plantar longitudinal arch of Virtual Chinese Human after plantar ligamentous structure failures. Clin Biomech 2007; 22:221-229. 
363

364

365

366

367

368

369

370

371

372

373

374

375

376

377

378

379

380

381

382

383

384

385

386

387

[18] J.T.M. Cheung, M. Zhang, A.K.L. Leung, Y.B. Fan, Three-dimensional finite element analysis of the foot during standing — a material sensitivity study, J. Biomech. 38 (2005) 10451054.

[19] A. Gefen, M. Megido-Ravid, Y. Itzchak, M. Arcan, Biomechanical analysis of the threedimensional foot structure during gait: a basic tool for clinical applications, J. Biomech. Eng-T Asme. 122 (2000) 630-639.

[20] S. Nakamura, R. Crowninshield, R. Cooper, An analysis of soft tissue loading in the foot--a preliminary report, Bull. Prosthet. Res. 10 (1981) 27-34.

[21] Y. Gu, X. Ren, J. Li, M.J. Lake, Q. Zhang, Y. Zeng. Computer simulation of stress distribution in the metatarsals at different inversion landing angles using the finite element method, Int. Orthop. 34 (2010) 669-676.

[22] S. Siegler, J. Block, C.D. Schneck, The mechanical characteristics of the collateral ligaments of the human ankle joint, Foot \& Ankle. 8 (1988) 234-242.

[23] D. Wright, D. Rennels, A Study of the Elastic Properties of Plantar Fascia, JBJS. 46 (1964) $482-492$.

[24] T.X. Qiu, E.C. Teo, Y.B. Yan, W. Lei, Finite element modeling of a 3D coupled foot-boot model, Med. Eng. Phys. 33 (2011) 1228-1233.

[25] A. Simkin, Structural analysis of the human foot in standing posture. Ph.D. Thesis, Tel Aviv University, Tel Aviv, Israel, 1982.

[26] K. A. Kirby, What future direction should podiatric biomechanics take? Clin Podiatr Med Sur. 18 (2001) 719-724.

[27] E. Morales-Orcajo, J. Bayod, E.B. de Las Casas, Computational foot modeling: scope and applications, Arch. Comput. Method. E. 23 (2016) 389-416.

[28] Y. Wang, D.W.C. Wong, M. Zhang, Computational models of the foot and ankle for pathomechanics and clinical applications: a review, Ann. Biomed Eng. 44 (2016) 213-221. 
388

389

390

391

392

393

394

395

396

397

398

399

400

401

402

403

404

405

406

407

408

409

410

411

412

413

[29] D.W.C. Wong, M. Zhang, A.K.L. Leung, First Ray Model Comparing Normal and Hallux

Valgus Foot, in: Zhang M, Fan Y (Eds.), Computational Biomechanics of the Musculoskeletal System, CRC Press, 2014, pp. 49-60.

[30] D.L. Camacho, W.R. Ledoux, E.S. Rohr, B.J. Sangeorzan, R.P. Ching, A threedimensional, anatomically detailed foot model: a foundation for a finite element simulation and means of quantifying foot-bone position, J. Rehabil Res. Dev. 39 (2002) 401.

[21] S.E. Benzley, E. Perry, K. Merkley, B. Clark, G. Sjaardama, A comparison of all hexagonal and all tetrahedral finite element meshes for elastic and elasto-plastic analysis, in Proceedings, 4th International Meshing RoundtableSandia National Laboratories Albuquerque, 1995.

[32] J.T.M. Cheung, M. Zhang, K.N.An, Effects of plantar fascia stiffness on the biomechanical responses of the ankle-foot complex. Clin Biomech. 19 (2004) 839-846.

[33] W.M. Glasoe, D.J. Nuckley, P.M. Ludewig, Hallux valgus and the first metatarsal arch segment: a theoretical biomechanical perspective, Phys. Ther. 90 (2010) 110.

[34] J.T. Deland, H. Sung, The medial crosssover toe: a cadaveric dissection, Foot Ankle. Int. 21 (2000) 375-378.

[35] C. Piqué-Vidal, M.T. Solé, J. Antich, Hallux valgus inheritance: pedigree research in 350 patients with bunion deformity, J. Foot Ankle Surg. 46 (2007) 149-154.

[36] E. Morales-Orcajo, J. Bayod, E.B. de Las Casas, Computational foot modeling: scope and applications. Arch Comput Methods Eng. 23 (2016) 389-416.

Fig. 1. Hallux valgus angle (HVA) and intermetatarsal angle (IMA) for hallux valgus foot model (a) and normal foot model (b).

Fig. 2. The three-dimensional finite element model and the application of boundary and loading conditions. 
414 Fig. 3. Comparison of the plantar pressure between experimental measurement $(\mathrm{a}, \mathrm{c})$ and 415 computational prediction (b, d) in balanced standing position.

416 Fig. 4. Comparison of the von-Mises stress at five metatarsals between normal foot and hallux

417 valgus foot.

418 Fig. 5. Comparison of contact pressure at metatarsophalangeal joints between normal foot and 419 hallux valgus foot.

420 Fig. 6. Posterior view of contact pressure at the first metatarsophalangeal joint of normal foot (a) 421 and hallux valgus foot (b). The red rectangles indicate the first MTP joint and the red circles 422 indicate the regions with high pressure (Unit: MPa).

423 Fig. 7. Comparison of the resultant joint force at the first metatarsophalangeal between normal 424 foot $\left(\mathrm{F}_{\mathrm{N}}\right)$ and hallux valgus foot $\left(\mathrm{F}_{\mathrm{S}}\right) . \mathrm{X}$ : Medial-Lateral direction (+: lateral; -: medial); Y: 425 Anterior-Posterior direction (+: anterior; -: posterior); Z: Superior-Inferior direction (+: inferior; $426-$-: superior). 
428 Table 1. Material properties and mesh element types for the foot model components.

\begin{tabular}{|c|c|c|c|c|}
\hline \multirow{2}{*}{ Component } & \multirow[b]{2}{*}{ Element Type } & Young's Modulus & \multirow{2}{*}{ Poisson's Ratio $v$} & Cross-section \\
\hline & & $\mathrm{E}(\mathrm{MPa})$ & & Area (mm2) \\
\hline Bone $[19,20]$ & Hexahedral solid & 7300 & 0.3 & - \\
\hline $\begin{array}{l}\text { Cartilage[16, } \\
\text { 21] }\end{array}$ & Hexahedral solid & 1 & 0.4 & \\
\hline $\begin{array}{l}\text { Ligaments[21, } \\
22]\end{array}$ & Tension-only spar & 260 & 0.4 & \\
\hline $\begin{array}{l}\text { Plantar } \\
\text { Fascia[21, 23] }\end{array}$ & Tension-only spar & 350 & 0.4 & 58.6 \\
\hline Plate[21] & Hexahedral solid & 17000 & 0.4 & - \\
\hline
\end{tabular}

429

430

431

432

433

434

435

436

437

438

439

440

441

442

443 
444 Table 2. The element type and coefficients of the hyperelastic material used for the encapsulated 445 soft tissue.
Element
C10
C01
$\mathrm{C} 20$
C11
$\mathrm{C} 02$
D1
D2

Type

\begin{tabular}{llllllll}
\hline Hexahedral & 0.08556 & -0.05841 & 0.03900 & -0.02319 & 0.00851 & 3.65273 & 0.00000
\end{tabular}

solid[24]

446 


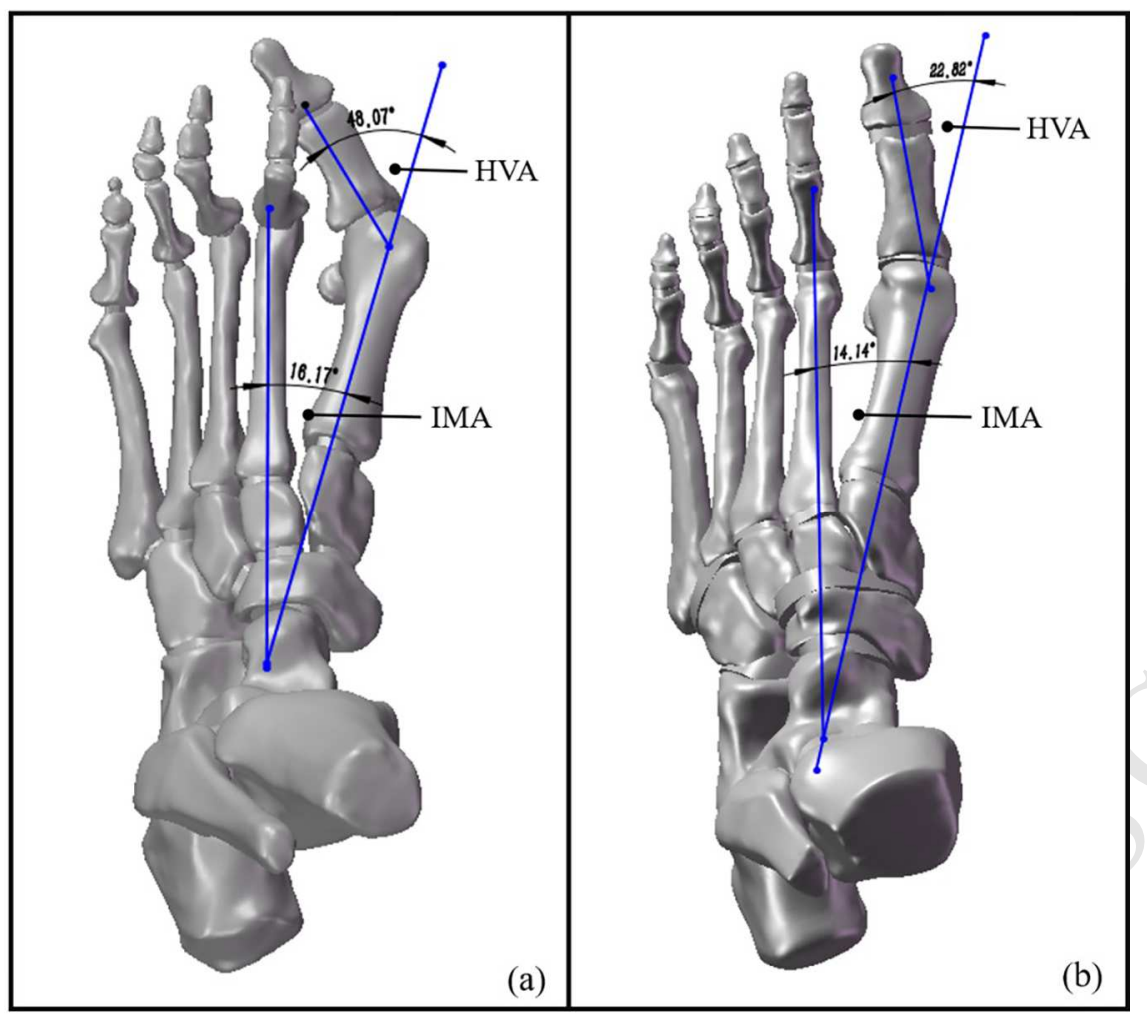



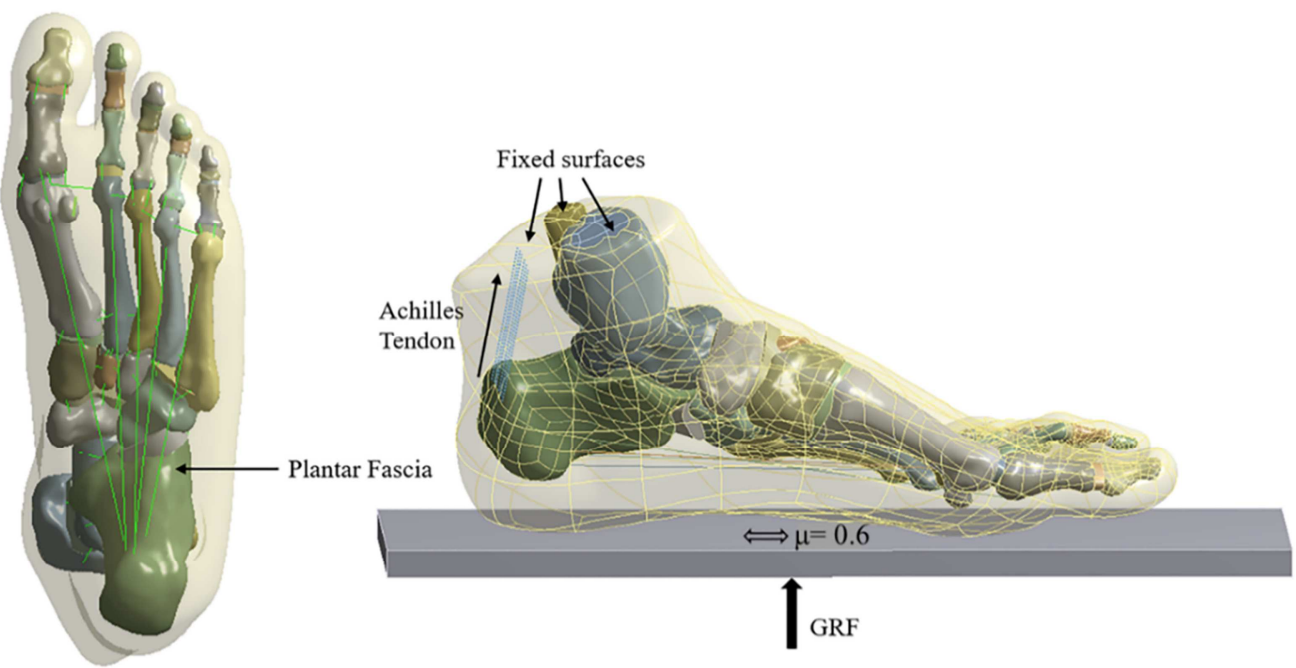

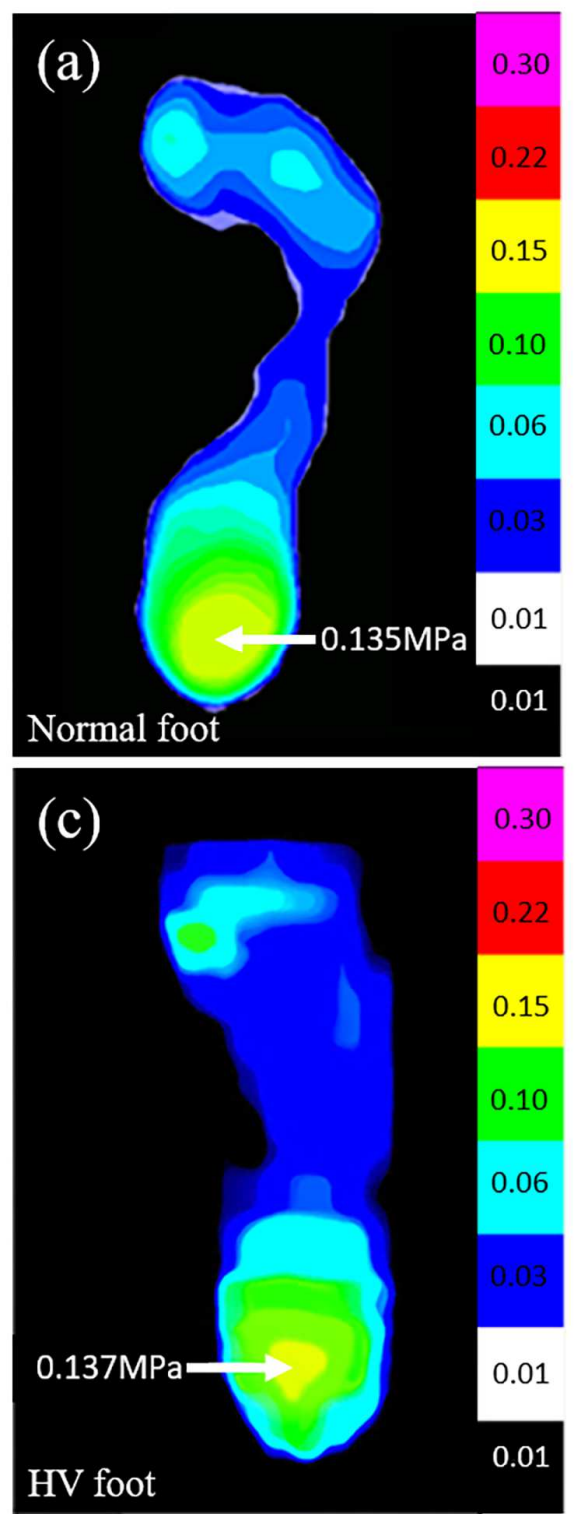
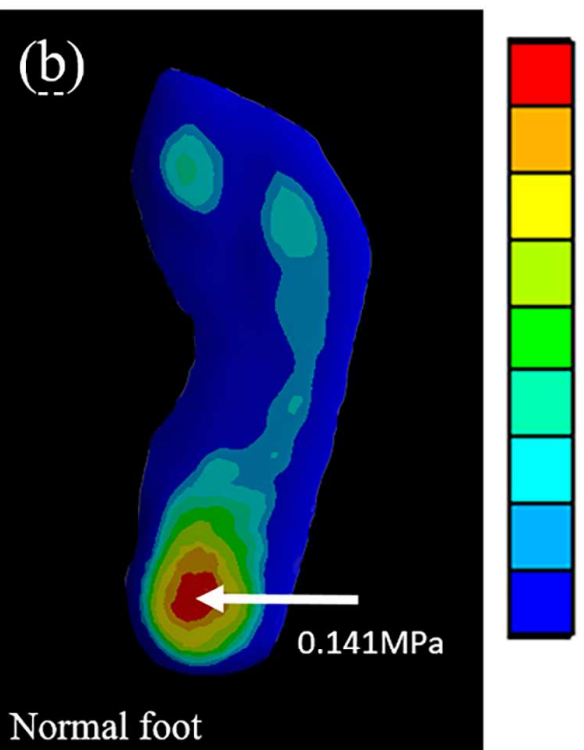

0.14084 Max

0.12519

0.10954

0.093891

0.078242

0.062594

0.046945

0.031297

0.015648

0 Min

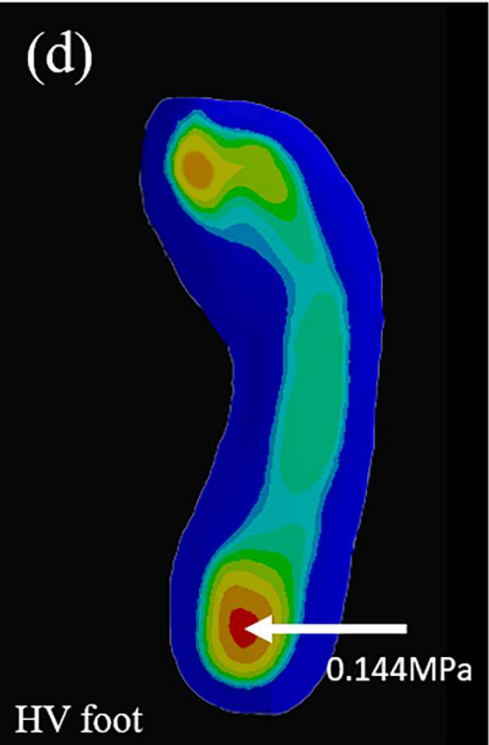

0.14435 Max

0.12831

0.11227

0.09623

0.080192

0.064153

0.048115

0.032077

0.016038

0 Min 


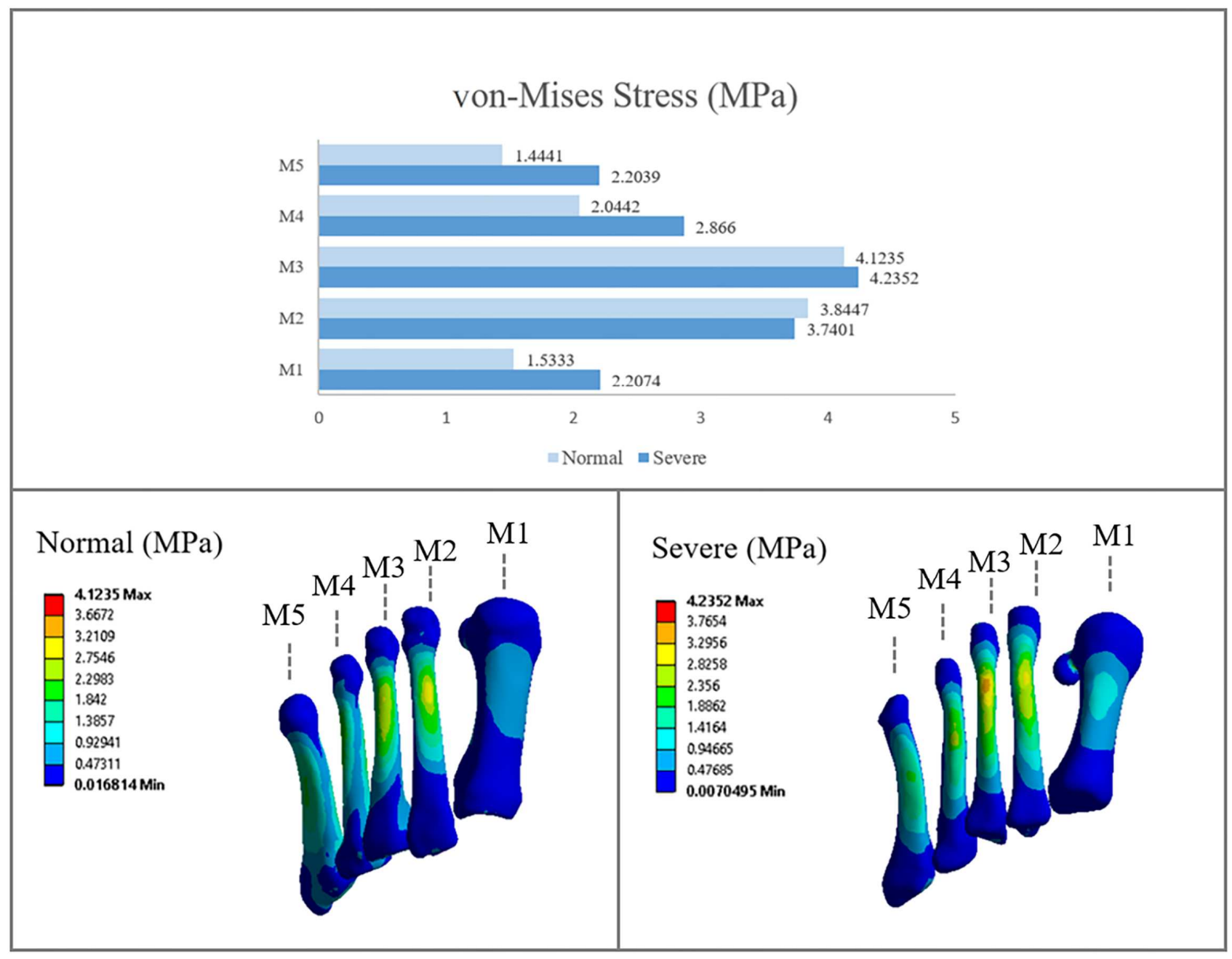




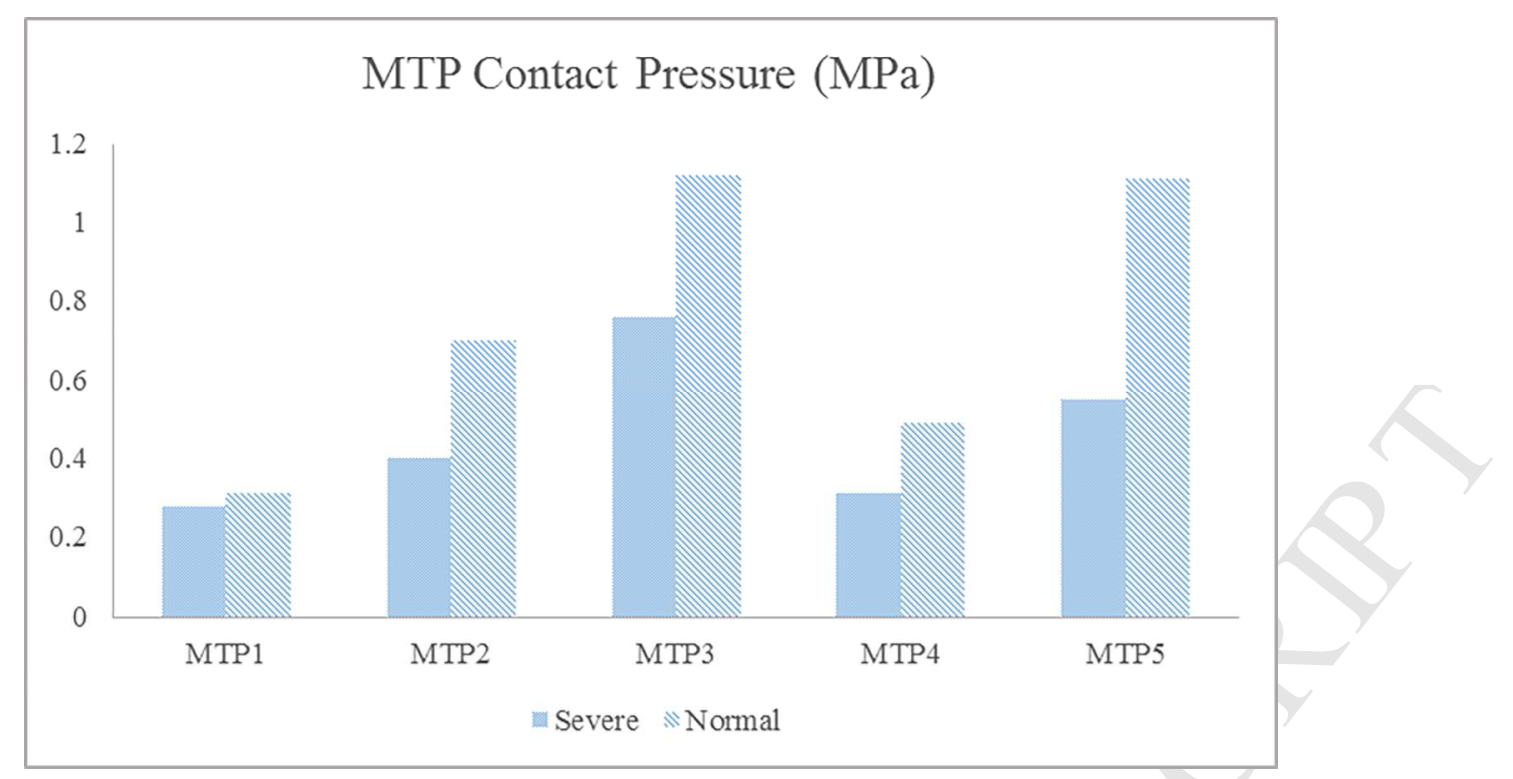




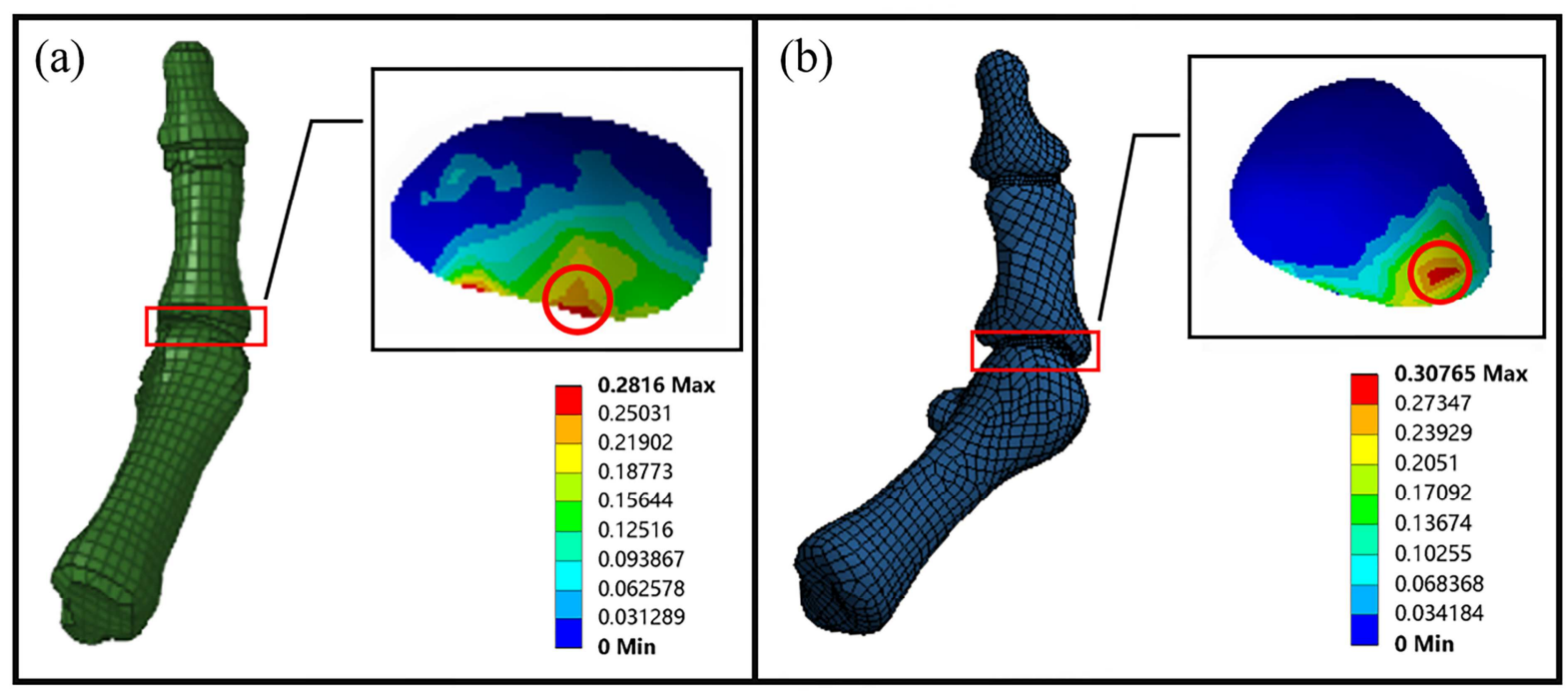




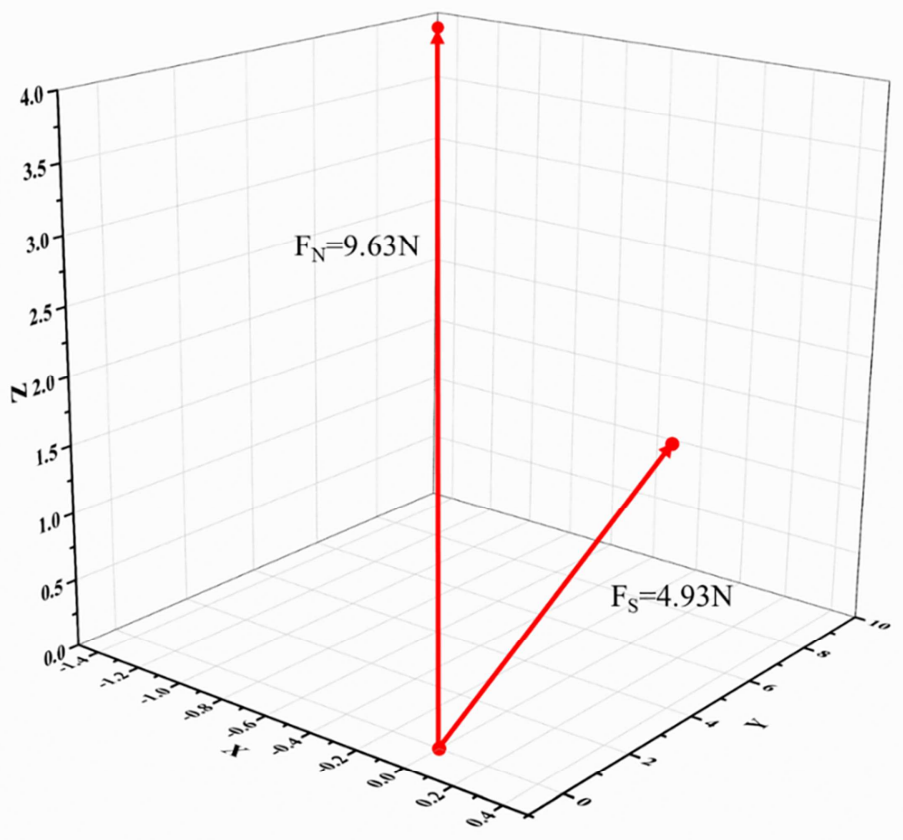


1. A severe hallux valgus FE models were constructed. 2. Pathological model predicted higher metatarsal stress and lower metatarsophalangeal pressure. 3 . Severe hallux valgus is a potential risk of forefoot pain and functional impairment. 